\title{
Subconscious Ratings of Inappropriate Coauthorship in Physics
}

\author{
Eugen Tarnow*
}

18-11 Radburn Road, Fair Lawn, NJ 07410, USA

\begin{abstract}
In the largest and most detailed survey on the ethics of scientific coauthorship to date, members of the American Physical Society (APS) were asked to judge the number of appropriate coauthors on their last published papers in three different ways [1]. The papers reported on by the respondents were papers in which the respondents' authorship was more involved than chance would dictate. From this discrepancy it is possible to infer "subconscious" rates of inappropriate authorship. The shapes of the curves of these subconscious rates are very similar to the curves of the consciously reported inappropriate authorship though the actual rates are much higher. The earlier reported "conscious" ratings indicated that the probability of any third and subsequent coathors being inappropriate was $23 \%$ for the APS guideline, $67 \%$ for the tighter guideline of the International Committee of Medical Journal Editors', or 59\% if the guideline was "direct contributions to scientific discovery or invention"; the currently reported subconscious rates varies between 94 and $97 \%$.
\end{abstract}

Key Words: Authorship, credit, physics, intellectual property, subconscious rating, inferred rating.

\section{INTRODUCTION}

Appropriate authorship assignment is of considerable importance to both scientists and to the public. Public recognition of intellectual achievement in terms of authorship is a very strong incentive for scientists to perform their work. Also, knowing who did a particular piece of work is important - scientists can contact the appropriate colleague, ask questions and obtain data -, and the public can shift funds to better scientists and optimize its return on investment in the scientific "market". Authorship is not an obvious state of affairs - in the world of business individual contributions are known only to the immediate colleagues; obtaining credit is less important, money is more important and inter- or even intra-company communication between people with similar interests is much more difficult.

Appropriate authorship assignment is also one of the most sensitive issues in science, with one of the most senior figures in science commenting that it is still in the pre-sixties stage of sex [1]. Interestingly, the earlier results indicated that there is a group of people who seems to strongly defy rating any authors on the byline as inappropriate [1].

No previous survey [1-7] checked reported rates of inappropriate authorship against a "real" measure. A comprehensive way of checking reported rates of inappropriate authorship against reality might be to perform in-depth interviews with all authors on a statistical number of bylines and then judge those reported contributions, with some way of resolving differing independent accounts, according to a chosen guideline. Another way is to trick the survey respondents into giving up the information without being aware that they did, which is what was done in the current survey.

\section{METHOD}

The content and method of the survey was described earlier [1] so here we satisfy ourselves with the most rudimentary

*Address correspondence to this author at the 18-11 Radburn Road, Fair Lawn, NJ 07410 USA; Tel: (201) 791-3220; E-mail: etarnow@avabiz.com details. The survey was produced using an internet survey system. Survey invitations were emailed to all APS members who had email accounts. The final return rate was $16 \%$ (4302 surveys). I did not perform a secondary survey of the non-responders but I examined the quality of the data two ways. First, I compared the age demographics obtained with publicly available US physics Ph.D graduation numbers corrected by US 1998 life tables of male death rates and found excellent qualitative and good quantitative agreement. Second, I studied the time dependence of the survey results: If some particular group of people would not respond to the survey at all, it seems reasonable that there should be some group of respondents with somewhat similar views who would answer the survey but with more hesitation than other respondents and thus make the survey results time dependent. The survey results were not time dependent and I argued that it was unlikely that it misrepresented such groups.

The statistics reported in this article refer to papers which "report a discovery or invention".

\section{RESULTS \& DISCUSSION}

\section{Coauthorship - Appropriateness of Actual Authorship}

Three conscious estimates of appropriateness of stated authorship were used [1]. Two authoritative authorship definitions included the APS guideline (question 3) and guideline of the International Committee of Medical Journal Editors (ICMJE, question 4). A third definition limited authorship to those authors who contributed "directly" to the "scientific discovery or invention" (question 7, the phrase in quotation marks is taken from the Nobel definition of why Nobelists get prizes). This question did not appear in a definition format.

\section{Coauthorship - Conscious Estimates of Inappropriate Coauthorship}

Fig. (1) shows various measures of the number of inappropriate coauthors (papers with 10 or more coauthors were combined into bins with at least 30 respondents). The curves 
were fit starting with the function probability that the nth coauthor is inappropriate $=a^{*}\left(1-e^{(-n / b)}\right)(1)$ where $a$ and $b$ are the fitting parameters. For $n>b$ the probability that the nth coauthor is inappropriate becomes a and otherwise the probability is somewhere between 0 and a. Using (1), the functional form for the percentage of inappropriate coauthorship shown in Fig. (1) is percentage of inappropriate coauthorship $=\mathrm{a}\left(\mathrm{n}-\mathrm{e}^{(-1 / \mathrm{b})}\left(1-\mathrm{e}^{(-\mathrm{n} / \mathrm{b})}\right) /\left(1-\mathrm{e}^{(-1 / b)}\right)\right) / \mathrm{n}(2)$.

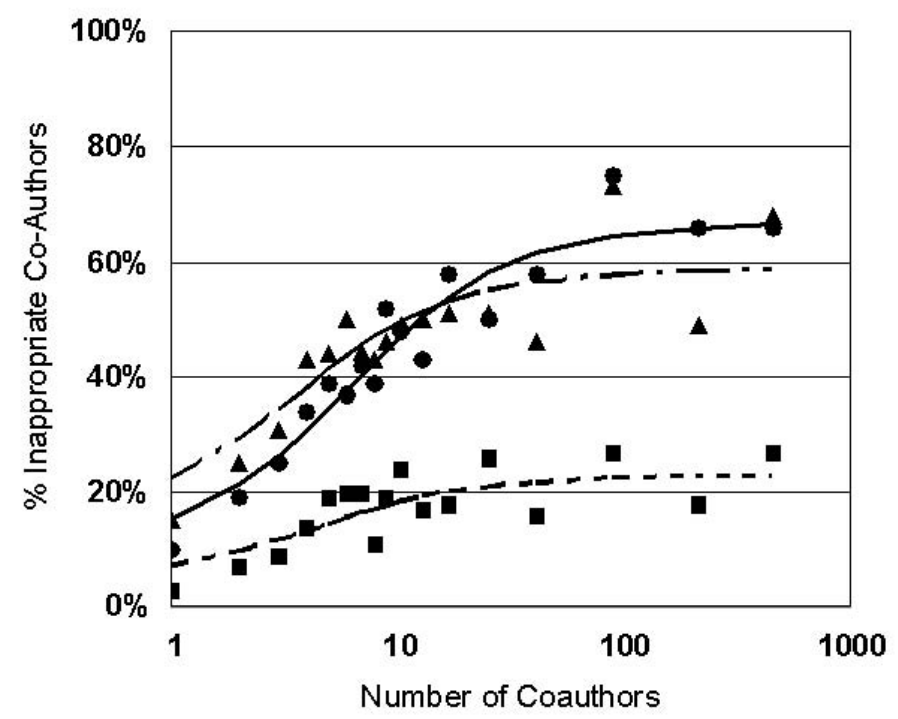

Fig. (1). Inappropriate authorship on all papers reporting discoveries measured three different ways. APS guideline is shown in filled squares and dashed line, ICMJE in filled circles and full line, the direct criterion in filled upward-pointing triangles and dash-dotted line.

The values of a (b) are 23\% (2.6) for the APS guideline, $67 \%$ (3.8) for ICMJE, and 59\% (2.1) for direct contributions.

\section{Coauthorship - Unconscious Estimates of Inappropriate Coauthorship}

Inferred estimates of "forgotten" authorship were obtained from a paper sample bias. This sample bias resulted from authors not including the correct paper in the survey. They had been asked to include "the most recent paper published in a refereed journal with yourself as an author." Once I asked whether the author was the most important author (question 10) and once I asked whether the author was the person to write down the initial authorship list (question 12). If the respondents correctly remembered THE PAPER, then on a paper with $\mathrm{N}$ authors they would answer yes to question 12 on the average $1 / \mathrm{N}$ times. The correct answer to question 10 is only somewhat less apparent - respondents may feel that more than one author was "the most important." The deviation of both sets of responses from $1 / \mathrm{N}$ gives us two additional measures of "forgotten" coauthorship. The inverse of the average answer defines the "remembered" authorship and "forgotten" coauthorship then becomes (N-1/average)/N.

The resulting "subconscious" rates of inappropriate authorship are shown in Fig. (2). They can be fit with the same method as the conscious inappropriate authorship and the two fitting factors a (b) become $94 \%$ (2.2) for the inferred author list measure and $97 \%$ (2.1) for the inferred measure of most important contributor.

A definite interpretation of the "subconscious" rates of inappropriate coauthorship will have to await future research. The current results show that it has the same functional form as inappropriate authorship suggests that the two are somehow connected. Because it is an inferred measure it probably failed to elicit an emotional response. This makes "forgotten" co-authorship complementary to inappropriate authorship. The pro of the actual judgments is that a conscious judging process is taking place, the pro of the inferred

\section{APPENDIX A: RELEVANT PARTS OF QUESTIONNAIRE}

In this questionnaire, THE PAPER will refer to the most recent paper published in a refereed journal with yourself as an author.

\section{How many authors are there in total on THE PAPER?}

3. In your opinion, how many of the authors on THE PAPER fulfill the requirements for authorship as defined by the APS: "Authorship should be limited to those who have made a significant contribution to the concept, design, execution or interpretation of the research study. All those who have made significant contributions should be offered the opportunity to be listed as authors. Other individuals who have contributed to the study should be acknowledged, but not identified as authors" [8].

4. In your opinion, how many of the authors on THE PAPER fulfill the requirements for authorship as defined by the BIOMEDICAL JOURNAL EDITORS:

"Authorship credit should be based only on 1) substantial contributions to conception and design, or acquisition of data, or analysis and interpretation of data; 2) drafting the article or revising it critically for important intellectual content; and 3) final approval of the version to be published. Conditions 1, 2, and 3 must all be met. Acquisition of funding, the collection of data, or general supervision of the research group, by themselves, do not justify authorship."

7. In your opinion, how many of the authors on THE PAPER contributed DIRECTLY to the scientific discovery or invention presented? (If THE PAPER did not report a discovery or invention, please enter 0 ).

\section{Were you the most significant contributor on THE PAPER?}

12. Were you the person who wrote down the initial version of the authorship list on THE PAPER? 
judgments is the exact opposite - that this conscious process, likely to be emotional, is probably not taking place.

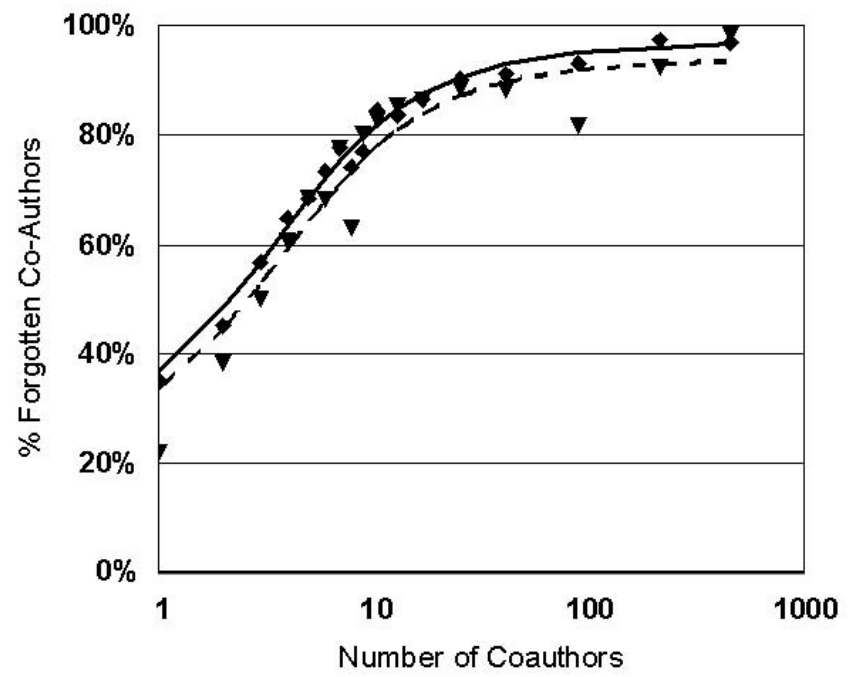

Fig. (2). Forgotten authorship on all papers reporting discoveries inferred from "most important author" result in triangles and dashed line and inferred from the "first person to write down the list" in diamonds and full line.

\section{ACKNOWLEDGMENT}

I would like to extend my gratitude for the time given to me by the survey respondents; for help with construction of survey questions and critical readings of the manuscript by Charles DeLeone, Michelle Fine and Rafi Kleiman.

\section{REFERENCES}

[1] Tarnow E. Coauthorship in physics. Sci Eng Ethics 2002; 8: 175190.

[2] Vasta R. The matter of publication credit: a survey of APA members. J Suppl Abstr Serv Catalog Sel Doc Psychol 1981; 11: 2-3.

[3] Swazey JP, Anderson MS, Louis KS. Ethical problems in academic research. Am Sci 1993; 81: 542-554.

[4] Kalichman M, Friedman P. A pilot study of biomedical trainees perceptions concerning research ethics. Acad Med 1992; 67: 767773.

[5] Eastwood S, Derish P, Leash E, Ordway S. Ethical issues in Biomedical Research: Perceptions and practices of postdoctoral research fellows responding to a survey. Sci Eng Ethics 1996; 2: 89114.

[6] Tarnow E. The Authorship List in Science: Junior physicists' perceptions of who appears and why. Sci Eng Ethics 1999; 5: 73-88.

[7] Yank V, Rennie D. Disclosure of researcher contributions: A study of original research articles. Ann Int Med 1999; 130: 661-70.

[8] Uniform requirement for manuscripts submitted to biomedical journals. International Committee of Medical Journal Editors. J Am Med Assoc 1997; 277: 927-934 